\title{
Lagrangian vector field and Lagrangian formulation of partial differential equations
}

\author{
M.Chen \\ Vanier College, 821 Ste-Croix Avenue, \\ St. Laurent, Québec, Canada H4L 3X9
}

Received November 4, 2004

In this paper we consider the Lagrangian formulation of a system of second order quasilinear partial differential equations. Specifically we construct a Lagrangian vector field such that the flows of the vector field satisfy the original system of partial differential equations.

Key words: Euler-Lagrange equations, vector fields, quasi-linear partial differential equations

PACS: $45.20 . J j, 02.30 . J r$

\section{Introduction}

Variational principle has played a fundamental role in the foundation of mathematics and physics. Minimization of an action integral by means of a Lagrange function gives rise to the set of Euler-Lagrange equations (ELs). Invariance of the action integral or ELs is intimately related to conservation laws [1]. It is well known in Lagrangian mechanics that the flows of the Lagrangian vector field satisfy ELs [2], where the set of ELs form a system of second order ordinary differential equations. In this paper we consider the Lagrangian formulation of a system of second order quasilinear partial differential equations in terms of ELs. Our objective is to construct a Lagrangian vector field $X_{\mathrm{L}}$ such that the flows of $X_{\mathrm{L}}$ satisfy ELs. We believe that this work is still lacking in the literature.

Let $B_{n}$ be an open set in $E_{n}$ with smooth boundary $\partial B_{n}$, and let $x=\left(x^{1}, x^{2}\right.$, $\left.\ldots, x^{n}\right)$ be the coordinate cover of $B_{n}$. Consider the following system of second order quasilinear PDEs:

$$
F_{a b}^{i j}\left(x, f^{c}, \partial_{k} f^{c}\right) \partial_{i} \partial_{j} f^{b}+G_{a}\left(x, f^{c}, \partial_{k} f^{c}\right)=0
$$

where $a, b, c \in[1,2, \ldots, N]$ and $\left\{F_{a b}^{i j}, G_{a}\right\}$ are given functions. In (1) we have adopted the conventional summation notation for repeated indices. The solution set of (1) for the $N$ functions $f^{a}$ can be viewed as a map $\Phi$ from the base space $B_{n}$ to the graph 
space $G=B_{n} \times R_{N}$ with coordinate cover $\left(x, u^{a}\right), 1 \leqslant a \leqslant N$. The map $\Phi$ is said to be a regular map if $\Phi^{*} \mu \neq 0$, where $\Phi^{*}$ is the pull back map of $\Phi, \mu=\mathrm{d} x^{1} \wedge \mathrm{d} x^{2} \wedge \ldots \wedge \mathrm{d} x^{n}$ is the volume element of $B_{n}$, and $\wedge$ is the exterior product of differential forms [3]. Next we imbed the graph space $G$ in a larger space $M=G \times R_{n N}$ with coordinate cover $\left(x, u^{a}, y_{i}^{a}\right)$. We furnish $M$ with $N$ contact 1 -forms $w^{a}=\mathrm{d} u^{a}-y_{i}^{a} \mathrm{~d} x^{i}$, where $w^{a} \wedge\left(\mathrm{d} w^{a}\right)^{n} \neq 0$, and $\left(\mathrm{d} w^{a}\right)^{n+1}=0$. Here $(.)^{m}$ denotes the $m^{\text {th }}$ exterior power. Then we extend the map $\Phi$ from $B_{n}$ to $M$ under the conditions $\Phi^{*} \mu \neq 0$ and $\Phi^{*} w^{a}=0$. Since $\Phi^{*} w^{a}=\Phi^{*}\left(\mathrm{~d} u^{a}\right)-\Phi^{*}\left(y_{i}^{a}\right) \Phi^{*}\left(\mathrm{~d} x^{i}\right)=\mathrm{d} f^{a}-\Phi^{*}\left(y_{i}^{a}\right) \mathrm{d} x^{i}=0$, we have $\Phi^{*}\left(y_{i}^{a}\right)=\partial_{i} f^{a}$, that is, the pull back of $y_{i}^{a}$ from $M$ to $G$ is $\partial_{i} f^{a}$. In the following discussions we say that $\Phi: B_{n} \rightarrow M$ is a regular map if $\Phi^{*} \mu \neq 0$ and $\Phi^{*} w^{a}=0$. From geometric point of view, a regular map $\Phi$ has the representation $\Phi \mid x^{i}=x^{i}, u^{a}=f^{a}(x), y_{i}^{a}=\partial_{i} f^{a}(x)$. It defines an $n$-dimensional section of $M$.

Based on the system of PDEs in (1), we define the system of $n$-forms $\left\{E_{a}\right\}$,

$$
E_{a}=F_{a b}^{i j}\left(x, u^{c}, y_{k}^{c}\right) \mathrm{d} y_{i}^{b} \wedge \mu_{j}+G_{a}\left(x, u^{c}, y_{k}^{c}\right) \mu,
$$

where $\left.\left.\mu_{j}=\partial_{j}\right\lrcorner \mu,\right\lrcorner$ is the inner multiplication between the partial differential operator $\partial_{j}$ and the $n$-form $\mu$, such that, $\left.\partial_{j}\right\lrcorner \mathrm{d} x^{i}=\delta_{j}^{i}$. Then

$$
\Phi^{*} E_{a}=\left\{F_{a b}^{i j}\left(x, f^{c}, \partial_{k} f^{c}\right) \partial_{i} \partial_{j} f^{b}+G_{a}\left(x, f^{c}, \partial_{k} f^{c}\right)\right\} \mu .
$$

Hence regular maps $\Phi$ solve the system of PDEs (1) if and only if $\Phi^{*} E_{a}=0$. Therefore, instead of considering the system of PDEs (1), we concentrate on the set of $n$-forms $\left\{E_{a}\right\}$ and consider regular maps $\Phi$ such that $\Phi^{*} E_{a}=0$.

\section{Lagrangian formulation of PDEs}

The solution set $\left\{f^{a}(x)\right\}$ forms the states of the system of PDEs (1). Suppose the system has a smooth Lagrangian function $L\left(x, f^{a}(x), \partial_{i} f^{a}(x)\right)$. Define the action integral $A\left[f^{a}\right]$ of the system by

$$
A\left[f^{a}\right]=\int_{B_{n}} L\left(x, f^{a}(x), \partial_{i} f^{a}(x)\right) \mu .
$$

Lift the Lagrangian function $L$ from the graph space $G$ to $M$ so that $L\left(x, u^{a}, y_{i}^{a}\right)$ is defined on $M$. Then $\Phi^{*} L\left(x, u^{a}, y_{i}^{a}\right)=L\left(x, f^{a}(x), \partial_{i} f^{a}(x)\right)$. We also define the Euler-Lagrange $n$-forms $E_{a}(L)$ by

$$
E_{a}(L)=L_{u^{a}} \mu-\left(\mathrm{d} L_{y_{i}^{a}}\right) \wedge \mu_{i}
$$

which can be rewritten as

$$
E_{a}(L)=L_{u^{a}} \mu-\left\{\left(L_{y_{i}^{a} u^{b}}\right) \mathrm{d} u^{b}+\left(L_{y_{i}^{a} y_{j}^{b}}\right) \mathrm{d} y_{j}^{b}\right\} \wedge \mu_{i} .
$$

Here $L_{y_{i}^{a} u^{b}}=\partial^{2} L /\left(\partial u^{b} \partial y_{i}^{a}\right), L_{y_{i}^{a} y_{j}^{b}}=\partial^{2} L /\left(\partial y_{j}^{b} \partial y_{i}^{a}\right)$. Assume that $L$ is independent of $x$ explicitly. Further assume that the values of $\left\{f^{a}(x)\right\}$ are specified on the boundary $\partial B_{n}$. It can be proved that a regular map $\Phi: B_{n} \rightarrow M$ stationarizes the action 
integral $A\left[f^{a}\right]$ if and only if $\Phi^{*} E_{a}(L)=0$ at every interior point of $B_{n}$ [4]. Hence $\Phi^{*} E_{a}(L)=0$ yields the Euler-Lagrange equations

$$
\frac{\partial L}{\partial f_{a}}=D_{i}\left[\frac{\partial L}{\partial\left(\partial_{i} f^{a}\right)}\right], \quad 1 \leqslant a \leqslant N,
$$

where

$$
D_{i}=\left(\partial_{i} f^{b}\right) \frac{\partial}{\partial f^{b}}+\left(\partial_{i} \partial_{j} f^{b}\right) \frac{\partial}{\partial\left(\partial_{j} f^{b}\right)}
$$

is the total derivative operator.

Now we consider the existence as well as the construction of the Lagrangian function $L$ on $M$. To this end, we let $V_{a}=G_{a}, \mathrm{~d} V_{a}^{i}=-F_{a b}^{i j} \mathrm{~d} y_{j}^{b}$, and $Q_{a}=V_{a} \mu-$ $\mathrm{d} V_{a}^{i} \wedge \mu_{i}$. The system $\left\{Q_{a}\right\}$ is said to admit a variational principle if and only if there exists a Lagrangian function $L\left(x, u^{a}, y_{i}^{a}\right)$ defined on $M$ such that $V_{a}=L_{u^{a}}$ and $V_{a}^{i}=L_{y_{i}^{a}}$. Set $Q=V_{a} \mathrm{~d} u^{a}+V_{a}^{i} \mathrm{~d} y_{i}^{a}$. Then $Q=\mathrm{d} L$ and $Q \wedge \mu=\mathrm{d}(L \mu)$ is a closed $(n+1)$-form. On the other hand, if $Q \wedge \mu$ is a closed $(n+1)$-form, it can be proved that the system $\left\{Q_{a}\right\}$ admits a variational principle [5]. Thus we first define $Q=V_{a} \mathrm{~d} u^{a}+V_{a}^{i} \mathrm{~d} y_{i}^{a}$ and determine whether or not $Q \wedge \mu$ is a closed $(n+1)$ form. Suppose the answer is affirmative. The Lagrange function $L$ can be written as $L=V_{a} u^{a}+V_{a}^{i} y_{i}^{a}$. Then $E_{a}(L)=Q_{a}$.

On the other hand, suppose the system $\left\{Q_{a}\right\}$ does not admit a variational principle. We can extend the space $M$ to a larger space $\hat{M}=M \times E_{N+n N}$ with coordinate cover $\left(x, u^{a}, y_{i}^{a}, \hat{u}^{a}, \hat{y}_{i}^{a}\right)$. Equip $\hat{M}$ with the contact 1 -forms $w^{a}=\mathrm{d} u^{a}-y_{i}^{a} \mathrm{~d} x^{i}$ and $\hat{w}^{a}=\mathrm{d} \hat{u}^{a}-\hat{y}_{i}^{a} \mathrm{~d} x^{i}$. In addition to the $n$-forms $Q_{a}$, we introduce $N$ auxiliary $n$-forms $\hat{Q}_{a}=\hat{V}_{a} \mu-\mathrm{d} \hat{V}_{a}^{i} \wedge \mu_{i}$, where $\hat{V}_{a}$ and $\hat{V}_{a}^{i}$ are given by

$$
\begin{aligned}
\hat{V}_{a} & =\partial_{a}\left(V_{b} \hat{u}^{b}+V_{b}^{j} \hat{y}_{j}^{b}\right), \\
\hat{V}_{a}^{i} & =\partial_{a}^{i}\left(V_{b} \hat{u}^{b}+V_{b}^{j} \hat{y}_{j}^{b}\right)
\end{aligned}
$$

with $\partial_{a}=\partial / \partial u^{a}$ and $\partial_{a}^{i}=\partial / \partial y_{i}^{a}$.

Let $L=V_{b} \hat{u}^{b}+V_{b}^{j} \hat{y}_{j}^{b}$. Define the 1 -form $Q$ for the extended system $\left\{Q_{a}, \hat{Q}_{a}\right\}$ by

$$
Q=V_{a} \mathrm{~d} \hat{u}^{a}+V_{a}^{i} \mathrm{~d} \hat{y}_{i}^{a}+\hat{V}_{a} \mathrm{~d} u^{a}+\hat{V}_{a}^{i} \mathrm{~d} y_{i}^{a} .
$$

Then $Q \wedge \mu=\mathrm{d}(L \mu)$. Hence the extended system $\left\{Q_{a}, \hat{Q}_{a}\right\}$ admits a variational principle with the Lagrangian function $L=V_{a} \hat{u}^{a}+V_{a}^{i} \hat{y}_{i}^{a}$. The Euler-Lagrange equations for the extended system can be written as

$$
\begin{aligned}
& \Phi^{*} Q_{a}=\Phi^{*}\left\{L_{u^{a}}-\left(L_{y_{i}^{a} \hat{u}_{b}}\right) \hat{y}_{i}^{b}-\left(L_{y_{i}^{a} \hat{y}_{j}^{b}}\right) \partial_{i} \hat{y}_{j}^{b}\right\} \mu=0, \\
& \Phi^{*} \hat{Q}_{a}=\Phi^{*}\left\{L_{\hat{u}^{a}}-\left(L_{\hat{y}_{i}^{a} u^{b}}\right) y_{i}^{b}-\left(L_{\hat{y}_{i}^{a} y_{j}^{b}}\right) \partial_{i} y_{j}^{b}\right\} \mu=0 .
\end{aligned}
$$

\section{Construction of the Lagrangian vector field}

In the following discussions we assume that $L$ is independent of $x$ explicitly. In Lagrangian Mechanics, the Lagrangian 1-form $\theta$, the Lagrangian 2-form $\Omega$, and the total energy of the system $E$ are defined as follows [6]: 


$$
\begin{aligned}
\theta & =\sum_{i} L_{\dot{q}^{i}} \mathrm{~d} q^{i}, \\
\Omega & =L_{\dot{q}^{i} q^{j}} \mathrm{~d} q^{i} \wedge \mathrm{d} q^{j}+L_{\dot{q}^{i} \dot{q}^{j}} \mathrm{~d} \dot{q}^{i} \wedge \mathrm{d} q^{j}, \\
E & =\dot{q}^{i} L_{\dot{q}^{i}}-L .
\end{aligned}
$$

In a similar manner, we define the Lagrangian 1-form $\theta_{\mathrm{L}}$, the Lagrangian 2 -form $\Omega_{\mathrm{L}}$, and the energy density $E_{\mathrm{L}}$ for the extended system $\left\{Q_{a}, \hat{Q}_{a}\right\}$ by

$$
\begin{aligned}
\theta_{\mathrm{L}}= & \sum_{i}\left[L_{y_{i}^{a}} \mathrm{~d} u^{a}+L_{\hat{y}_{i}^{a}} \mathrm{~d} \hat{u}^{a}\right], \\
\Omega_{\mathrm{L}}= & \mathrm{d} \theta_{\mathrm{L}}=\sum_{i}\left\{\left(L_{y_{i}^{a} \hat{y}_{j}^{b}}\right) \mathrm{d} \hat{y}_{j}^{b} \wedge \mathrm{d} u^{a}+\left(L_{y_{i}^{a} \hat{u}^{b}}\right) \mathrm{d} \hat{u}^{b} \wedge \mathrm{d} u^{a}\right. \\
& \left.\quad+\left(L_{\hat{y}_{i}^{a} y_{j}^{b}}\right) \mathrm{d} y_{j}^{b} \wedge \mathrm{d} \hat{u}^{a}+\left(L_{\hat{y}_{i}^{a} u^{b}}\right) \mathrm{d} u^{b} \wedge \mathrm{d} \hat{u}^{a}\right\}, \\
E_{\mathrm{L}}= & y_{i}^{a} L_{y_{i}^{a}}+\hat{y}_{j}^{b} L_{\hat{y}_{j}^{b}}-L .
\end{aligned}
$$

We assume that the Lagrangian function $L$ is nondegenerate, i.e., $L_{y_{i}^{a} \hat{y}_{j}^{b}}, L_{\hat{y}_{i}^{a} y_{j}^{b}}$ are nowhere vanishing on $\hat{M}$. Since $L, \Omega_{\mathrm{L}}$ and $E_{\mathrm{L}}$ are independent of $x$, we define the Lagrangian vector field $X_{\mathrm{L}} \in T \hat{M}^{1}$ by

$$
X_{\mathrm{L}}=Y_{k}^{c} D_{k u^{c}}+\hat{Y}_{k}^{b} D_{k \hat{u}^{b}}+Z_{k l}^{c} D_{k y_{l}^{c}}+\hat{Z}_{k l}^{c} D_{k \hat{y}_{l}^{c}},
$$

where $Y_{k}^{c}, \hat{Y}_{k}^{b}, Z_{k l}^{c}, \hat{Z}_{k l}^{c}$ are functions defined on $\hat{M}$, while $D_{k u^{c}}, D_{k \hat{u}^{b}}, D_{k y_{l}^{c}}, D_{k \hat{y}_{l}^{c}}$ are partial differentiation operators with respect to $u^{c}, \hat{u}^{b}, y_{l}^{c}, \hat{y}_{l}^{c}$ respectively. Notice that we have adopted double subscripts for the $D$ operators to emphasize that the flows of $X_{\mathrm{L}}$ are given by

$$
Y_{k}^{c}=\frac{\partial u^{c}}{\partial t^{k}}, \quad \hat{Y}_{k}^{b}=\frac{\partial \hat{u}^{b}}{\partial t^{k}}, \quad Z_{k l}^{c}=\frac{\partial y_{l}^{c}}{\partial t^{k}}, \quad \hat{Z}_{k l}^{c}=\frac{\partial \hat{y}_{l}^{c}}{\partial t^{k}},
$$

where $\left(t^{1}, t^{2}, \ldots, t^{n}\right)$ are $n$ parameters.

In Lagrangian mechanics the Lagrangian vector field $X$ is constructed by the condition $X\lrcorner \Omega=-\mathrm{d} E$. It is well known that the flows of $X$ satisfy the EulerLagrange equations [6]. In a similar manner, we determine the Lagrangian vector field $X_{\mathrm{L}}$ by the condition

$$
\left.X_{\mathrm{L}}\right\lrcorner \Omega_{\mathrm{L}}=-\mathrm{d} E_{\mathrm{L}} .
$$

By comparing the coefficients of $\mathrm{d} y_{i}^{a}, \mathrm{~d} \hat{y}_{j}^{b}, \mathrm{~d} u^{a}$ and $\mathrm{d} \hat{u}^{b}$ on both sides of (12) we can obtain the following results:

$$
\begin{aligned}
& -Y_{i}^{a}\left(L_{y_{i}^{a} \hat{y}_{j}^{b}}\right)=-y_{i}^{a}\left(L_{y_{i}^{a} \hat{y}_{j}^{b}}\right), \\
& -\hat{Y}_{j}^{b}\left(L_{\hat{y}_{j}^{b} a_{i}^{a}}\right)=-\hat{y}_{j}^{b}\left(L_{\hat{y}_{j}^{b} y_{i}^{a}}\right), \\
& \left(L_{y_{i}^{a} \hat{u}^{b}} \hat{Y}_{i}^{b}+\left(L_{y_{i}^{a} \hat{y}_{j}^{b}}\right) \hat{Z}_{i j}^{b}=L_{u^{a}},\right. \\
& \left(L_{\hat{y}_{j}^{b} u^{a}}\right) Y_{j}^{a}+\left(L_{\hat{y}_{j}^{b} y_{i}^{a}}\right) Z_{j i}^{a}=L_{\hat{u}^{b}} .
\end{aligned}
$$

\footnotetext{
${ }^{1}$ Alternatively, we can write

$$
X_{\mathrm{L}}=W_{i} \partial_{i}+Y_{k}^{c} D_{k u^{c}}+\hat{Y}_{k}^{b} D_{k \hat{u}^{b}}+Z_{k l} D_{k y_{l}^{c}}+\hat{Z}_{k l}^{c} D_{k \hat{y}_{l}^{c}} .
$$
}

However, $W_{i}$ turns out to be zero identically. 
Since the Lagrangian function is nondegenerate, by (13a) and (13b) we obtain $Y_{i}^{a}=$ $y_{i}^{a}, \hat{Y}_{j}^{b}=\hat{y}_{j}^{b}$. On the other hand, $\hat{Z}_{i j}^{b}$ and $Z_{j i}^{a}$ can be solved from (13c) and (13d) respectively. In view of (11) we can identify $t^{k}=x^{k}$. Thus, $Z_{j i}^{a}=\partial_{j} y_{i}^{a}, \hat{Z}_{i j}^{b}=\partial_{i} \hat{y}_{j}^{b}$, and (13c), (13d) become

$$
\begin{aligned}
\left(L_{y_{i}^{a} \hat{u}^{b}}\right) \hat{y}_{i}^{b}+\left(L_{y_{i}^{a} \hat{y}_{j}^{b}}\right)\left(\partial_{i} \hat{y}_{j}^{b}\right) & =L_{u}^{a}, \\
\left(L_{\hat{y}_{j}^{b} u^{a}}\right) y_{j}^{a}+\left(L_{\hat{y}_{j}^{b} y_{i}^{a}}\right)\left(\partial_{j} y_{i}^{a}\right) & =L_{\hat{u}^{b}} .
\end{aligned}
$$

Hence (14a) and (14b) in conjunction with (11) give rise to (8a) and (8b). Therefore the flows of $X_{\mathrm{L}}$ satisfy the Euler-Lagrange equations.

To conclude this section we consider an example in extended irreversible thermodynamics:

$$
\partial_{x}^{2} T-\frac{\tau}{\chi} \partial_{t}^{2} T-\frac{1}{\chi} \partial_{t} T=0
$$

where $T$ is the temperature, $\tau$ and $\chi$ are constants.

Let $x^{1}=x, x^{2}=t$. Then $y_{1}=\partial_{1} T, y_{2}=\partial_{2} T, \mu=\mathrm{d} x \wedge \mathrm{d} t=\mathrm{d} x^{1} \wedge \mathrm{d} x^{2}, \mu_{1}=$ $\left.\left.\partial_{1}\right\lrcorner \mu=\mathrm{d} x^{2}, \mu_{2}=\partial_{2}\right\lrcorner \mu=-\mathrm{d} x^{1}$. The contact manifold $M$ has the global coordinate cover $\left(x^{1}, x^{2}, u, y_{1}, y_{2}\right)$, and is equipped with the contact 1 -form $\omega=\mathrm{d} u-y_{i} \mathrm{~d} x^{i}$, $i=1,2$.

(i) By (15) the 2-form $E$ is given by

$$
E=-\mathrm{d}\left(\frac{1}{\chi} u+\frac{\tau}{\chi} y_{2}\right) \wedge \mu_{2}+\mathrm{d} y_{1} \wedge \mu_{1}
$$

The regular map $\Phi: B_{2} \rightarrow M$ yields $\Phi^{*} E=0$, which is identical to (15). Let $Q_{1}=E=v_{1} \mu-\mathrm{d} v_{1}^{i} \wedge \mu_{i}$. We have $v_{1}=0, v_{1}^{1}=-y_{1}, v_{1}^{2}=\frac{1}{\chi}\left(u+\tau y_{2}\right)$. If we set $Q=v_{1} \mathrm{~d} u+v_{1}^{1} \mathrm{~d} y_{1}+v_{1}^{2} \mathrm{~d} y_{2}$, then $Q \wedge \mu$ is not a closed 3-form. Consequently (15) does not admit a variational principle ${ }^{2}$. For this reason we enlarge $M$ to $\hat{M}$, where $\hat{M}$ has the coordinate cover $\left(x, u, y_{1}, y_{2}, \hat{u}, \hat{y}_{1}, \hat{y}_{2}\right)$, and is equipped with the contact 1 -forms $\omega=\mathrm{d} u-y_{i} \mathrm{~d} x^{i}, \hat{\omega}=\mathrm{d} \hat{u}-\hat{y}_{i} \mathrm{~d} x^{i}$.

(ii) Let

$$
L=v_{1} \hat{u}+v_{1}^{1} \hat{y}_{1}+v_{1}^{2} \hat{y}_{2}=-y_{1} \hat{y}_{1}+\left(\frac{1}{\chi} u+\frac{\tau}{\chi} y_{2}\right) \hat{y}_{2} .
$$

\footnotetext{
${ }^{2}$ It should be noted that there are many different formulations for a system of second order quasi-linear PDEs to be transcribed into a system on $n$-forms. For the specific PDE in (15), we could have constructed the 2 -form$$
E^{\prime}=\mathrm{d} y_{1} \wedge \mu_{1}-\frac{\tau}{\chi} \mathrm{d} y_{2} \wedge \mu_{2}-\frac{1}{\chi} y_{2} \mu
$$

In view of the of the contact 1 -form $\omega=\mathrm{d} u-y_{i} \mathrm{~d} x^{i}$, we can easily show that

$$
E=E^{\prime}-\frac{1}{\chi} \omega \wedge \mu_{2}
$$

i.e., $E^{\prime}=E \bmod \omega$. Hence both $\Phi^{*} E=0$ and $\Phi^{*} E^{\prime}=0$ give rise to (15). Similarly, if we set $v_{1}=-\chi^{-1} y_{2}, \mathrm{~d} v_{1}^{1}=-\mathrm{d} y_{1}, \mathrm{~d} v_{1}^{2}=\tau \chi^{-1} \mathrm{~d} y_{2}$, then $Q_{1}^{\prime}=v_{1} \mu-\mathrm{d} v_{1}^{i} \wedge \mu_{i}=Q_{1}+\chi^{-1} \omega \wedge \mu_{2}$. Hence $Q_{1}^{\prime}=E^{\prime}$ and $Q_{1} \wedge \mu=Q_{1}^{\prime} \wedge \mu$.
} 
Then

$$
\hat{v}_{1}=\partial_{u} L=\frac{1}{\chi} \hat{y}_{2}, \hat{v}_{1}^{1}=L_{y_{1}}=-\hat{y}_{1}, \hat{v}_{1}^{2}=L_{y_{2}}=\frac{\tau}{\chi} \hat{y}_{2}
$$

Thus

$$
\hat{Q}_{1}=\hat{v}_{1} \mu-\mathrm{d} \hat{v}_{1}^{i} \wedge \mu_{i}=\frac{1}{\chi} \hat{y}_{2} \mu+\mathrm{d} \hat{y}_{1} \wedge \mu_{1}-\frac{\tau}{\chi} \mathrm{d} \hat{y}_{2} \wedge \mu_{2}
$$

Set

$$
Q=v_{1} \mathrm{~d} \hat{u}+v_{1}^{1} \mathrm{~d} \hat{y}_{1}+v_{1}^{2} \mathrm{~d} \hat{y}_{2}+\hat{v}_{1} \mathrm{~d} u+\hat{v}_{1}^{1} \mathrm{~d} y_{1}+\hat{v}_{1}^{2} \mathrm{~d} y_{2} .
$$

Then $Q=\mathrm{d} L$. Hence $Q \wedge \mu=\mathrm{d}(L \mu)$ and, the extended system $\left\{Q_{1}, \hat{Q}_{1}\right\}$ admits a variational principle with the Lagrange function $L$ given by

$$
L=-y_{1} \hat{y}_{1}+\frac{\tau}{\chi} y_{2} \hat{y}_{2}+\frac{1}{\chi} u \hat{y}_{2} .
$$

(iii) Based on the Lagrange function $L$, we can construct the Euler-Lagrange 2forms as follows:

$$
\begin{aligned}
& E(L)=L_{u} \mu-\mathrm{d} L_{y_{i}} \wedge \mu_{i}=\frac{1}{\chi} \hat{y}_{2} \mu+\mathrm{d} \hat{y}_{1} \wedge \mu_{1}-\frac{\tau}{\chi} \mathrm{d} \hat{y}_{2} \wedge \mu_{2}=\hat{Q}_{1}, \\
& \hat{E}(L)=L_{\hat{u}} \mu-\mathrm{d} L_{\hat{y}_{i}} \wedge \mu_{i}=\mathrm{d} y_{1} \wedge \mu_{1}-\frac{\tau}{\chi} \mathrm{d} y_{2} \wedge \mu_{2}-\frac{1}{\chi} \mathrm{d} u \wedge \mu_{2}=Q_{1} .
\end{aligned}
$$

Hence the Euler-Lagrange equations for the extended system $\left\{Q_{1}, \hat{Q}_{1}\right\}$ become

$$
\begin{aligned}
\Phi^{*} E(L)= & \Phi^{*}\left\{L_{u}-\left(L_{y_{i} \hat{u}}\right) \hat{y}_{i}-\left(L_{y_{i} \hat{y}_{j}}\right) \partial_{i} \hat{y}_{j}\right\} \mu \\
= & \Phi^{*}\left\{\frac{1}{\chi} \hat{y}_{2}+\partial_{1} \hat{y}_{1}-\frac{\tau}{\chi} \partial_{2} \hat{y}_{2}\right\} \mu=0, \\
\Phi^{*} \hat{E}(L)= & \Phi^{*}\left\{L_{\hat{u}}-\left(L_{\hat{y}_{i} u}\right) y_{i}-\left(L_{\hat{y}_{i} y_{j}}\right) \partial_{i} y_{j}\right\} \mu \\
= & \Phi^{*}\left\{\frac{1}{\chi} y_{2}-\partial_{1} y_{1}+\frac{\tau}{\chi} \partial_{2} y_{2}\right\} \mu=0 .
\end{aligned}
$$

Equations (17a) and (17b) in turn yield

$$
\begin{aligned}
& \partial_{x}^{2} \hat{T}+\frac{1}{\chi} \partial_{t} \hat{T}-\frac{\tau}{\chi} \partial_{t}^{2} \hat{T}=0 \\
& \partial_{x}^{2} T-\frac{1}{\chi} \partial_{t} T-\frac{\tau}{\chi} \partial_{t}^{2} T=0
\end{aligned}
$$

(iv) By the Lagrangian function

$$
L=-y_{1} \hat{y}_{1}+\left(\frac{1}{\chi} u+\frac{\tau}{\chi} y_{2}\right) \hat{y}_{2}
$$

we construct the Lagrangian 1-form

$$
\theta_{\mathrm{L}}=\sum_{i}\left(L_{y_{i}} \mathrm{~d} u+L_{\hat{y}_{i}} \mathrm{~d} \hat{u}\right)=-\hat{y}_{1} \mathrm{~d} u+\frac{\tau}{\chi} \hat{y}_{2} \mathrm{~d} u-y_{1} \mathrm{~d} \hat{u}+\left(\frac{\tau}{\chi} y_{2}+\frac{1}{\chi} u\right) \mathrm{d} \hat{u} .
$$


Then the Lagrangian 2-form $\Omega_{\mathrm{L}}$ becomes

$$
\Omega_{\mathrm{L}}=\mathrm{d} \theta_{\mathrm{L}}=-\mathrm{d} \hat{y}_{1} \wedge \mathrm{d} u+\frac{\tau}{\chi} \mathrm{d} \hat{y}_{2} \wedge \mathrm{d} u-\mathrm{d} y_{1} \wedge \mathrm{d} \hat{u}+\frac{\tau}{\chi} \mathrm{d} y_{2} \wedge \mathrm{d} \hat{u}+\frac{1}{\chi} \mathrm{d} u \wedge \mathrm{d} \hat{u} .
$$

Define the Lagrangian vector field $X_{\mathrm{L}}$ by

$$
X_{\mathrm{L}}=Y_{i} D_{i u}+\hat{Y}_{i} D_{i \hat{u}}+Z_{i j} D_{i y_{j}}+\hat{Z}_{i j} D_{i \hat{y}_{j}}
$$

The flows of $X_{\mathrm{L}}$ can be written as

$$
\frac{\partial u}{\partial t^{i}}=Y_{i}, \quad \frac{\partial \hat{u}}{\partial t^{i}}=\hat{Y}_{i}, \quad \frac{\partial y_{j}}{\partial t^{i}}=Z_{i j}, \quad \frac{\partial \hat{y}_{j}}{\partial t^{i}}=\hat{Z}_{i j} .
$$

On the other hand, the energy density of the extended system $\left\{Q_{1}, \hat{Q}_{1}\right\}$ is given $b^{3}$

$$
E_{\mathrm{L}}=y_{i} L_{y_{i}}+\hat{y}_{i} L_{\hat{y}_{i}}-L=-y_{1} \hat{y}_{1}+\frac{\tau}{\chi} y_{2} \hat{y}_{2} .
$$

Now $X_{\mathrm{L}}$ can be determined by the equation $\left.X_{\mathrm{L}}\right\lrcorner \Omega_{\mathrm{L}}=-\mathrm{d} E_{\mathrm{L}}$. Hence

$$
\begin{aligned}
& Y_{i}=y_{i}, \quad \frac{\tau}{\hat{Y}_{i}}=\hat{y}_{\dot{i}}, \\
& \frac{\tau}{\chi} Z_{22}+\frac{1}{\chi} Y_{2}-Z_{11}=\frac{1}{\chi} \partial_{2} y_{2}+\frac{1}{\chi} y_{2}-\partial_{1}=0, \\
& \frac{\tau}{\chi} \hat{Z}_{22}-\frac{1}{\chi} \hat{Y}_{2}-\hat{Z}_{11}=\frac{\tau}{\chi} \partial_{2} \hat{y}_{2}-\frac{1}{\chi} \hat{y}_{2}-\partial_{1} \hat{y}_{1}=0 .
\end{aligned}
$$

Applying $\Phi^{*}$ on (19a) and (19b) we can recover (18a) and (18b) respectively. Hence the flows of $X_{\mathrm{L}}$ give rise to the Euler-Lagrange equations.

\section{Conclusions}

It is well known that many important theories in physical sciences can be described by ordinary or partial differential equations. In general these equations can be formulated via the variational principle in terms of a Lagrangian function. In this paper we consider the Lagrangian formulation of a system of quasilinear PDEs, where the underlying system of equations can be recast in terms of the Euler-Lagrange equations. Then we construct a vector field such that the flows of the vector field satisfy the Euler-Lagrange equations.

A similar approach to the problem considered in this paper has also been developed by Guo, Shang and Mei [7]. Based on the idea of adjoint symmetries, they modified a nonconservative system by adding a set of adjoint equations to the system. Thus the dimension of the configurational manifold is double of the dimension of the original system. Consequently the modified system admits a regular Lagrangian. From this point of view, there appears to be some similarity between our work and the work of Guo, et al. However, the methodology is very different, especially in the construction of the Lagrangian vector field. It would be interesting to extend this work to a wider class of PDEs, or to consider the Hamiltonian formulation of PDEs and the construction of the Hamiltonian vector field.

\footnotetext{
${ }^{3}$ We have abused the definition of the energy density $E_{\mathrm{L}}$ for the extended system $\left\{Q_{1}, \hat{Q}_{1}\right\}$. If $E_{\mathrm{L}}$ is given by $E_{\mathrm{L}}=y_{1} \hat{y}_{1}+\tau \chi^{-1} y_{2} \hat{y}_{2}$, then $\int_{B_{n}} E_{\mathrm{L}} \mu$ is a constant. But the determination of $X_{\mathrm{L}}$ by the condition $\left.X_{\mathrm{L}}\right\lrcorner \Omega_{\mathrm{L}}=-\mathrm{d} E_{\mathrm{L}}$ leads to contradiction.
} 


\section{Acknowledgements}

The author wishes to thank the reviewer for many valuable comments, in particular, for bringing reference [7] to the attention of the author.

\section{References}

1. Bluman G.W., Kumei J. Symmetry and Differential Equations. Springer-Verlag, New York, 1989; Olver P.J. Applications of Lie Groups to Differential Equations. SpringerVerlag, Berlin, 1993.

2. Libermann P., Marle C.-M. Symplectic Geometry and Analytic Mechanics. D. Reidel Publishing Company, Dordrecht, Holland, 1987.

3. Edelen D.G.B. Applied Exterior Calculus. John Wiley and sons, New York, 1985.

4. See [3], Chapter 7, p. 297.

5. See [3], Chapter 7, p. 301.

6. See [1], p. 74; Wasserman R.H. Tensors and Manifolds with Applications to Mechanics and Relativity, p. 277. Oxford University Press, 1992.

7. Guo Y.K., Shang M., Mei F.X., Int. J. Theoret. Phys., 1999, 38, 1017.

\section{Векторне поле Лагранжа та лагранжове формулювання парціальних диференціальних рівнянь}

\section{М.Чен}

Коледж Ваньє, Квебек, Канада

Отримано 4 листопада 2004 р.

В роботі розглядається лагранжове формулювання системи квазілінійних парціальних диференціальних рівнянь другого порядку. Зокрема, представлена конструкція векторного поля Лагранжа у формі, коли потік векторного поля задовільняє вихідні системи парціальних диференціальних рівнянь.

Ключові слова: рівняння Ейлера-Лагранжа, векторні поля, квазілінійні парціальні диференціальні рівняння

PACS: 45.20.Jj, 02.30.Jr 